\title{
Relationships Between the Expression of the ACTN3 Gene and Explosive Power of Soccer Players
}

\author{
by \\ Daria Domańska-Senderowska ${ }^{1}$ Paulina Szmigielska ${ }^{1}$, Aleksandra Snochowska ${ }^{1}$, \\ Zbigniew Jastrzębski², Anna Jegier, Justyna Kiszałkiewicz', Joanna Jastrzębska², \\ Dorota Pastuszak-Lewandoska ${ }^{1}$, Pawet Cięszczyk ${ }^{2,4}$, Aleksandra Suchanecka ${ }^{5}$, \\ Michat Wilk ${ }^{6}$, Michat Brzeziański ${ }^{1}$ Ewa Brzeziańska-Lasota ${ }^{1}$
}

Muscle strength and maximal speed are factors determining athlete's results during competition. Their association with ACTN3 gene activity has been documented. The purpose of this study was the analysis of ACTN3 gene expression during a 2 month training cycle of soccer players and its correlation with the countermovement jump (CMJ) and squat jump (SJ). The study group consisted of 22 soccer players (aged 17-18). The study material included peripheral blood lymphocytes. The relative expression $(R Q)$ of the ACTN3 gene was analyzed by $q P C R$ and performed before and after the two-month training cycle. Before the training cycle low expression levels of $A C T N 3$ (median $R Q=0.95$ ) were observed, yet after the training cycle they were elevated (median $R Q=1.98)(p=0.003)$. There was an increase in performance of both jumps: $S J(p=0.020)$ and $C M J(p=0.012)$ at the end of the training cycle. A simultaneous increase in the ACTN3 gene expression level and height in both jump tests was observed in $73 \%$ of athletes ( $p>0.05)$. There were no significant relationships between the ACTN3 gene expression level and the results of the CMJ and SJ. However, explosive strength is a complex feature shaped by many different factors and it could be the reason why we did not observe correlations between these variables.

Key words: $\alpha$-actinin-3 gene, soccer players, explosive power, squat jump, counter movement jump

\section{Introduction}

Soccer is a team sport which is well-known as an intermittent and high-intensity discipline. Apart from low- or moderate-intensity running, during a 90-min game strikers, midfielders, defenders and goal keepers perform short and long passes, sprints, accelerations, heading, jumping and tackling. The ability to perform these kinds of movements, with maximum effort (e.g. high speed) in a short period of time, is defined as explosive muscular strength. This property of skeletal muscles depends on efficient anaerobic and aerobic capacity and is recognized as a primary determinant of performance, especially in team sports relying on speed and power (Joo and Seo, 2016; Newton and Kraemer, 1994).

Physical performance is thought to be a complex trait shaped by many environmental variables such as training, diet, psychological willingness or social factors (Joo and Seo, 2016; Newton and Kraemer, 1994; MacArthur and North, 2007). In fact, the genetic background plays the first fiddle, determining performance-related

1 - Department of Biomedicine and Genetics, Medical University of Lodz, Lodz, Poland.

2 - Faculty of Physical Culture, Gdansk University of Physical Education and Sport, Gdansk, Poland.

3 - Department of Sport Medicine, Medical University of Lodz, Lodz, Poland.

4 - Independent Laboratory of Genetics and Molecular Biology, Military Institute of Hygiene Epidemiology, Warsaw, Poland.

5 - Independent Laboratory of Health Promotion, Pomeranian Medical University in Szczecin.

${ }^{6}$ - Institute of Sport Sciences, The Jerzy Kukuczka Academy of Physical Education, Katowice, Poland.

Authors submitted their contribution to the article to the editorial board.

Accepted for printing in the Journal of Human Kinetics vol. 69/2019 in September 2019. 
features; even muscle strength and muscle mass have been reported to be genetically conditioned (Eynon et al., 2013, 2014; Kikuchi et al., 2016; Kikuchi and Nakazato, 2015). Nevertheless, it is important to note that athletic performance is determined by multiple genes (Eynon et al., 2013; Szalata et al. 2019). Along with cardiac output and oxygen uptake, the appropriate distribution of fast and slow twitch fibers in muscles can define physical fitness (MacArthur and North, 2007). Explosive strength is a vital predictor for intensive physical effort and its heritability varies from 74 to 84\% (Eynon et al., 2013a). Since skeletal muscles, constituting approximately $33-40 \%$ of total body mass, are the greatest and the major site of the metabolism in the human body (Houweling et al., 2017), genes encoding its building particles (e.g. proteins) responsible for maintaining a proper structure and functioning have been considered to be important agents that affect athletic performance (Eynon et al., 2013a). Indeed, $\alpha$ actinin-3 gene (ACTN3) polymorphism was one of the first that evidenced the association between the genotype and athletic performance (Cieszczyk et al., 2012; Pimenta et al., 2013; Santiago et al., 2008; Yang et al., 2003; Kalinowski et al., 2019).

ACTN3 DNA coding sequence is located in the long arm of chromosome 11 (11q13-q14) (Fattahi and Najmabadi, 2012). The protein product of this gene, $\alpha$-actinin- 3 is a member of the $\alpha$-actinin proteins family and one of two isoforms expressed in human skeletal muscles (Cieszczyk et al., 2012; Fattahi and Najmabadi, 2012; Kikuchi and Nakazato, 2015). ACTN3 is a part of the Z-line in the sarcomere where it provides structural support during muscle contraction by anchoring actin filaments. It also participates in interactions with other muscle proteins and thereby acts as a signaling and metabolic molecule (Norman et al., 2009). The expression of ACTN3 is almost exclusively limited to type II (particularly the type IIb) fibers (Norman et al., 2009; Santiago et al., 2008). These fast, glycolytic muscle fibers produce explosive strength which is essential for generating fast movements during a soccer match (Fattahi and Najmabadi, 2012; Pimenta et al., 2013).

To date, over 200 polymorphisms affecting athletic performance have been described, but results are most consistent in case of ACTN3 R557X (rs1815739) (Eynon et al., 2013). Point mutation in a sequence of $A C T N 3$ results in conversion of an arginine (R) amino acid at the 577 residue into premature codon stop $(X)$. Individuals homozygous for a premature stop codon variant (577XX) are characterized by total lack of $\alpha$-actinin3 in muscles, whereas 577R allele is the proper, functional version of the ACTN3 (Cieszczyk et al., 2012; Fattahi and Najmabadi, 2012; MacArthur et al., 2007). It is estimated that $20 \%$ of the world population are carriers of the 577XX variant. High prevalence of the 577XX genetic variant may be an evolutionary gain since it exerts an protective effect during acute eccentric exercise (Eynon et al., 2014; Ivarsson and Westerblad, 2015).

Animal model studies have shown that owing to $\alpha$-actinin-3 deficiency, muscles of ACTN3 knockout mice were characterized by a decreased diameter of fast muscle fibers and reduced muscle mass as a consequence, slow muscle fibers-like properties (shift in the anaerobic and aerobic enzyme activity towards more oxidative pathway of metabolism) and increased endurance capacity when compared to the wild-type gene variant (Eynon et al., 2013). Vincent and colleagues (2007) established that in human muscle biopsy samples obtained from healthy, untrained young men, proportion of fast-twitch fibers differed among 577RR and 577XX individuals, in favor of 577RR carriers. Genotype 577XX seems to be beneficial for endurance performance; this genotype occurs with higher frequency in endurance athletes than in sprinting/power athletes. In terms of soccer players, those harboring 577RR genotype have higher jumping potential and are faster at short distances, whereas 577XX athletes have a more efficient aerobic capacity (Cieszczyk et al., 2012; Pimenta et al., 2013).

It is well proven that ACTN3 R577X polymorphism is associated with elite athlete status (Fattahi and Najmabadi, 2012; Kikuchi et al., 2016; MacArthur and North, 2007; MacArthur et al., 2007; Santiago et al., 2008; Holdys et al. 2011). Nevertheless, to the authors' best knowledge, the number of studies performed to characterize the ACTN3 gene expression level is very limited, especially with regard to soccer players. Therefore, the aim of our study was to evaluate whether there was a relationship between the ACTN3 expression level and explosive strength. Potential association could be a factor that should be taken into account during individual training-load regulation and match strategy planning. 


\section{Methods}

\section{Participants}

The study was approved by the Medical University of Lodz Ethics Committee (RNN/157/16/KE). All participants provided full written informed consent prior to study commencement.

The study group consisted of 22 male soccer players $(17.52 \pm 0.70 \mathrm{yr}, 178 \pm 0.70 \mathrm{~cm}, 68.05$ $\pm 9.18 \mathrm{~kg})$. All the participants were submitted to equivalent trial conditions that lasted two months (middle of April - middle of June, 2016). The physical activity involved endurance, speed and strength exercises arranged in different sequences during the week. The intensity of exercises was estimated by the heart rate (HR) that was equal to the anaerobic threshold (AnT), but did not exceed $90 \%$ of the HRmax. Besides, at the beginning of the experiment, maximal running intensity and the lactate threshold were determined for each of the participants (Radzimiński et al., 2010).

Training drills included: running intervals, technical and tactical drills, as well as speed, coordination, plyometric, aerobic and anaerobic activities. In addition, players attended smallsided games (conducted on a field with natural grass with $120 \mathrm{~m}^{2}$ per soccer player) and a match every Saturday. All the participants played 4 games, $4 \mathrm{~min}$ each with $3 \mathrm{~min}$ active rest.

\section{The CMJ/SJ test}

To estimate the explosive power of the players, the $\mathrm{CMJ} / \mathrm{SJ}$ tests were conducted. Before the test, participants performed a 20-min warm-up involving five vertical jumps. The test comprised two maximal vertical jumps without an arm swing (squat jump, SJ) and two with arm swings (countermovement jump, CMJ). In the squat jump, participants settled down in a full squatted posture with knees close together and maximally flexed. After that, the knees and hips were extended to jump vertically off the ground with arms resting on hips. A countermovement jump was performed from un upright standing position. The vertical jump was preceded by a downward movement (countermovement phase) until a full squatted posture with arms swinging back. In a propulsive phase of the jump, the knees and hips were extended and arms swinging upward. The rest interval between jumps was two minutes. The best (the highest) jump was used in subsequent analysis. All of the jumps were performed on a tensometric mat (Smart Jump Mat 120 x $120 \mathrm{~cm}$ - Fusion Sport, Cooper Plains, Australia 2012).

\section{Collection of biological material}

Blood samples were collected in a fasted state before the start and after the 2-month training cycle. Before the blood was aspirated, the players rested in the supine position for 10 minutes. Blood was collected into $5 \mathrm{ml}$ EDTA containing tubes, by inserting a needle into the antecubital vein with a short stasis.

A density gradient cell separation solution Histopaque®-1077 (Sigma-Aldrich Co.) was used for lymphocyte isolation.

Both the biological material (blood) and the jump height data were collected at two time points - before and after the 2-month training cycle ( $\mathrm{T}_{1}$ and $\mathrm{T}_{2}$, respectively).

\section{Gene expression analysis}

RNA isolation was carried out using a mirVana $^{\mathrm{TM}}$ miRNA Isolation Kit (Life Technologies, Carlsbad, CA, USA), according to the manufacturer's protocol. The quality and quantity of isolated RNA was assessed spectrophotometrically (Eppendorf BioPhotometrTM Plus, Eppendorf, Hamburg, Germany). The purity of total RNA (ratio of $16 S$ to $18 \mathrm{~S}$ fraction) was determined in the automated electrophoresis using the RNA Nano Chips Lab Chipplates in Agilent 2100 Bioanalyzer (Agilent Technologies, Santa Clara, CA). Complementary DNA (cDNA) was transcribed from $100 \mathrm{ng}$ of total RNA, using a High-Capacity cDNA Reverse Transcription Kit (Applied Biosystems, Carlsbad, CA, USA) in a total volume of $20 \mu \mathrm{l}$, according to the manufacturer's protocol. The relative expression analysis was performed in the $7900 \mathrm{HT}$ Fast Real-Time PCR System (Applied Biosystems, Carlsbad, CA, USA) using TaqMan probes for the studied gene ACTN3 (Hs00153812_m1) and ACTB gene (Hs99999903_m1) used as an endogenous control. The PCR mixture contained cDNA (1 to 100 ng), 20× TaqManRGene Expression Assay, 2× KAPA PROBE Master Mix (2x) ABI Prism Kit (Kapa Biosystems, Wilmington, MA, USA), and RNase-free water in a total volume of $20 \mu \mathrm{l}$. The expression levels (RQ values) of the studied gene were calculated using the delta CT method, with the adjustment to the $\beta$-actin expression level and in relation to the expression level of the calibrator, for which RQ value was equal to 1 . 


\section{Statistical Analysis}

The Shapiro - Wilk test was carried out to assess the normal distribution. The Wilcoxon signed rank test was used to compare the levels of relative expression values (RQs) and the jump height values at both time points. The Spearman's rank correlation coefficient was used to assess the correlation between gene relative expression levels and the jump height data at both time points. Outcomes of $p<0.05$ were considered statistically significant. Calculations were performed using Statistica for Windows 13.0.

\section{Results}

Relative expression levels of ACTN3 gene.

The relative expression level (RQ value) of $A C T N 3$ gene was studied at two time points before and after the training cycle $\left(T_{1}\right.$ and $T_{2}$, respectively). The median values were as follows: in $\mathrm{T}_{1}=0.95 \pm 1.21$ and in $\mathrm{T}_{2}=1.98 \pm 2.13$. The differences in $R Q$ values between the two time points were statistically significant $(p=0.003$; Wilcoxon signed rank test), with a higher gene expression level at $\mathrm{T}_{2}$ (Figure 1).

Statistical analysis of the differences in SJ height data $[\mathrm{cm}]$ between the two time points.

The median SJ height $[\mathrm{cm}]$ values were as follows: in $\mathrm{T}_{1}=33.35 \pm 0.78$ and in $\mathrm{T}_{2}=38.32 \pm 1.37$, average performance improvement was $11.32 \%$. The differences in SJ height between the two studied time points were statistically significant ( $p$ $=0.020$; Wilcoxon signed rank test), with higher $\mathrm{SJ}$ values in $\mathrm{T}_{2}$ (Figure 2).

Statistical analysis of the differences in CMJ height data [cm] between both studied time points.

The median CMJ height values $[\mathrm{cm}]$ were as follows: in $\mathrm{T}_{1}=39.63 \pm 2.27$ and in $\mathrm{T}_{2}=43.78 \pm 1.89$, average performance improvement was $10.89 \%$. The differences in CMJ height between the two studied time points were statistically significant ( $p$ $=0.012$; Wilcoxon signed rank test), with higher $\mathrm{CMJ}$ values in $\mathrm{T}_{2}$ (Figure 3).

Statistical analysis of the reciprocal relationship between the expression of the ACTN3 gene (median $R Q$ value) and the median jump height [cm] in the two studied time points.

Finally, we assessed the reciprocal relationship between the expression level of ACTN3 gene (median RQ values) and jump height (Spearman's rank correlation test). The results are presented in Table 1 . No statistically significant correlation was found.

\section{Discussion}

In the present study we observed, to our knowledge, for the first time, the increased expression levels of ACTN3 gene in the majority of the studied soccer players. Although there is a lot of research focused on the role of ACTN3 polymorphism, there is no research focused on ACTN3 gene expression in athletes. Thus, it is difficult to discuss the obtained results due to the lack of other published studies focused on ACTN3 gene expression.

The available data clearly show a significant association between ACTN3 genotype and performance in multiple cohorts of athletes (Eynon et al., 2013b; Papadimitrou et al., 2016). This mechanism is strongly supported by mechanistic data from the ACTN3 knockout mouse model (Kim et al., 2014). It has been shown that ACTN3 gene and protein levels change in response to exercise in female Sprague-Dawley rats (Kim et al., 2014). However, it is not clear whether the observed changes in ACTN3 expression are a direct result or the cause of adaptation to physical activity. The Ma et al.'s (2013) study clearly documented the association between ACTN3 polymorphism ( $\mathrm{R}$ allele) and power events. These findings suggest that polymorphic gene variants influence human physical performance.

ACTN3 gene highly affects muscle fiber composition, as it is responsible for the production of alpha-actinin-3, a protein that is only found in fast twitch muscle fibers (IIA et IIX). 


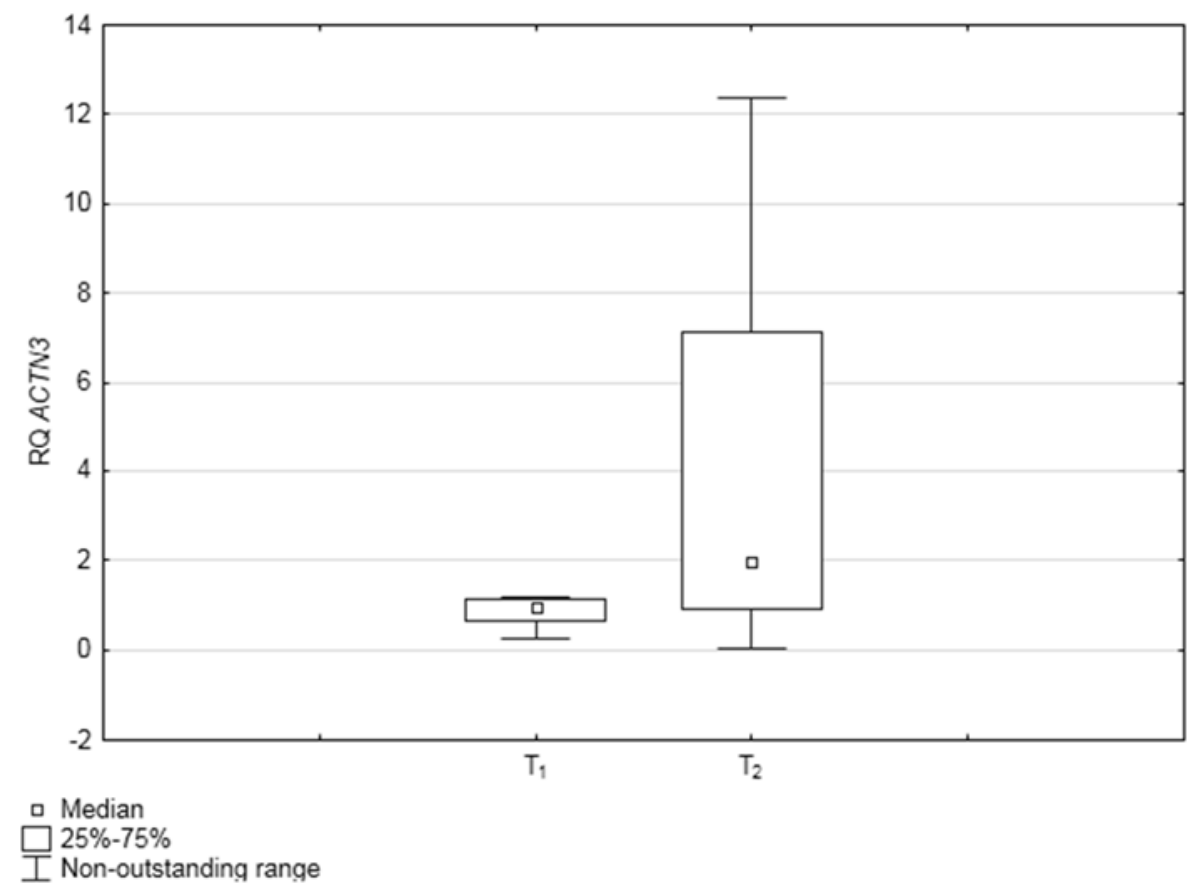

Figure 1

Box-and-whisker plots, representing ACTN3 expression levels (median RQ values) at the two time points: before and after the training cycle ( $T_{1}$ and $T_{2}$, respectively); $n=22$.

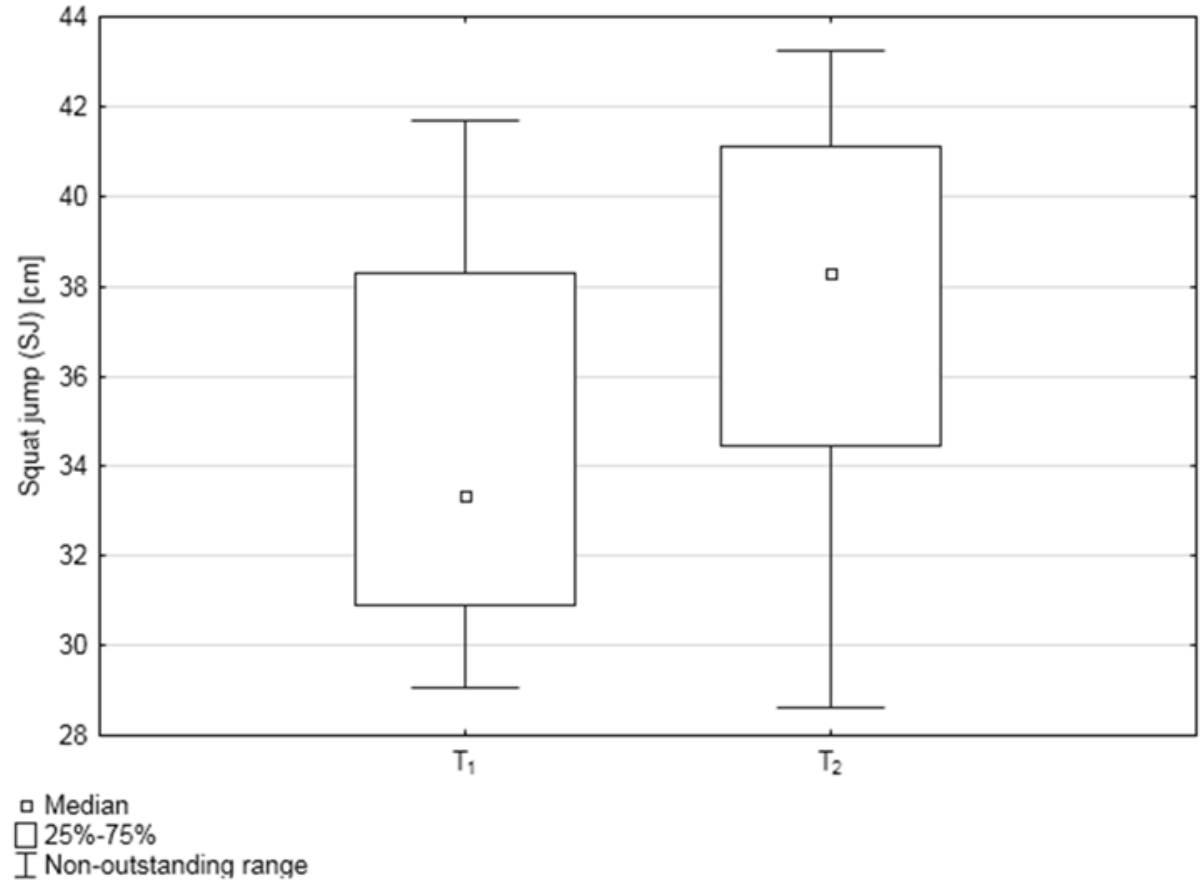

Figure 2

Box-and-whisker plots, representing median squat jump (SJ) values [cm] at the two studied time points: before and after the training cycle (T1 and $T_{2}$, respectively); $n=22$. 


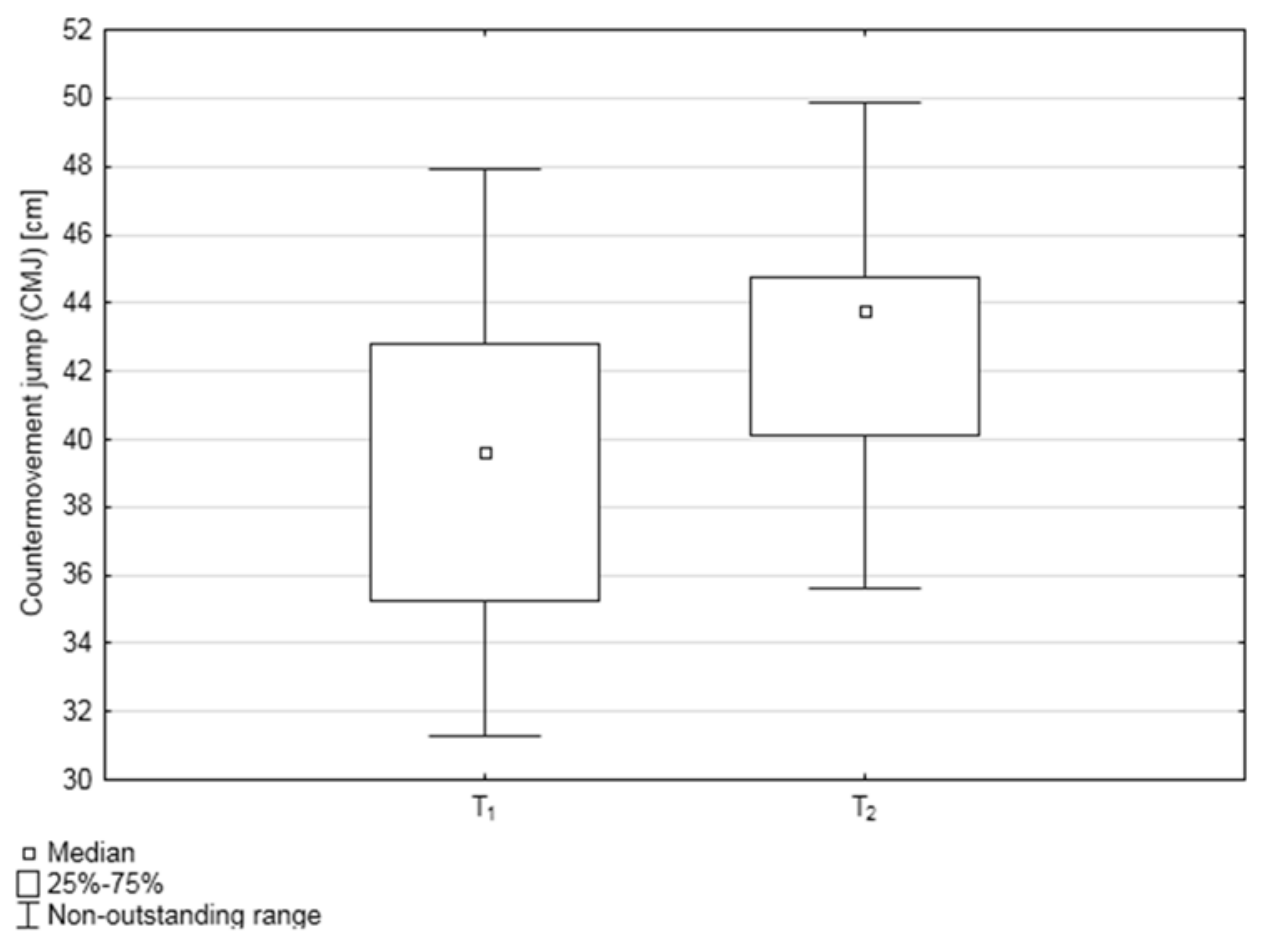

Figure 3

Box-and-whisker plots, representing median countermovement jump (CMJ) values [cm] in the two studied time points: before and after the training cycle $\left(T_{1}\right.$ and $T_{2}$, respectively); $n=22$.

Table 1

The results of statistical analysis regarding correlations between the ACTN3 gene expression level and jump height

\begin{tabular}{lll}
\hline & \multicolumn{2}{l}{ ACTN3 gene expression level } \\
\cline { 2 - 3 } & $\mathrm{T}_{1}$ & $\mathrm{~T}_{2}$ \\
\hline & rho $=-0.085$ & rho $=-0.258$ \\
CMJ & $p=0.705$ & $p=0.246$ \\
& & \\
& & rho $=-0.258$ \\
& rho $=-0.217$ & $p=0.247$
\end{tabular}

All $p$ and rho values were calculated with the Spearman's rank correlation coefficient. The reciprocal relationship between the expression level of ACTN3 gene (median RQ values) and jump height [cm] in two studied time points: before and after the training cycle ( $T_{1}$ and $T_{2}$, respectively); $n=22$. 
Moreover another study showed that roughly $18 \%$ of the world population are homozygous for a nonsense mutation (R577X) in ACTN3 deficiency (North et al., 1999). There are evolutionary changes in expression (decrease) of $\alpha$ actinin-3 (Ivarsson and Westerblad, 2015). Additionally, ACTN3 interacts with various genes that are associated with muscle remodeling and myofibrillar organization (Yang et al., 2003). Therefore, with regard to the above-mentioned facts, it seems that the evaluation of ACTN3 expression is potentially an important marker of adaptation to exercise in athletes. The assessment of ACTN3 expression and the relationship with explosive strength in athletes also seems to be an interesting factor.

Besides the increased gene expression level, we also observed increased explosive strength after a two month training cycle (measured with the SJ and CMJ) by $11.32 \%$ and $10.89 \%$, respectively (Table 1). Our training cycle was intensive and long-term. Similar results in soccer players, after the same training period, were also obtained by Loturco et al. (2016). On the other hand, other researchers examined explosive strength (CMJ and SJ) in soccer players under different training conditions. They observed the short-term effect of a lower weekly volume program (one session), as well as the effect of training-induced adaptations of different weekly training frequencies on both physiological and performance variables (SJ and CMJ) during the pre-season and throughout the in-season in soccer players (Silva et al., 2015). The research has shown that explosive strength, i.e., CMJ and SJ, sprint starts, improved during a soccer season by $2.49 \%$ and $6.4 \%$, respectively (Di Giminiani and Visca, 2017; Silva et al., 2015). Similar results have been reported in studies conducted on young elite English (Williams et al., 2011) and Serb soccer players (Mirkov et al., 2010), of whom jump performance (CMJ) increased by about $7 \%$.

It has been shown that differences observed in explosive strength also depend on the player's genotype. The athletes with ACTN3 RR genotypes showed $6 \%$ higher handgrip strength as compared to the XX group. They also jumped 5\% higher in both the SJ and CMJ tests (Broos et al., 2015). Additionally, ACTN3 RR and RX individuals presented better strength abilities than ACTN3 XX individuals. Inversely, the latter showed higher levels of endurance (Pimenta et al., 2013). In the study performed by Orysiak et al. (2014) youth male Polish athletes with ACTN3 RR genotypes had significantly greater explosive leg power in all jumping trials than those with the $X X$ genotype. Unfortunately, we did not perform genotype analysis of ACTN3 in our study, given the size of the group.

Reassuming, our study was the first, in which we showed increased ACTN3 mRNA levels in soccer players. Additionally, we assessed the presence of a correlation between the ACTN3 mRNA expression level and power. We documented that both study variables were significantly higher in all athletes after the 2 month training cycle.

Due to the size of the studied group, we did not perform genotype analysis and could not link it with the gene expression level. However, explosive strength is a complex feature shaped by many different factors and it could be the reason why we did not observe any relationship between these variables. There is a great need to replicate this study on a larger group of athletes in order to perform all the analysis necessary to fully understand the observed phenomenon.

\section{References}

Broos S, Van Leemputte M, Deldicque L, Thomis MA. History-dependent force, angular velocity and muscular endurance in ACTN3 genotypes. Eur J Appl Physiol, 2015; 115: 1637-1643

Cieszczyk P, Sawczuk M, Maciejewska-Karlowska A, Ficek K. ACTN3 R577X polymorphism in top-level Polish rowers. J Exerc Sci Fit, 2012; 10: 12-15

Di Giminiani R, Visca C. Explosive strength and endurance adaptations in young elite soccer players during two soccer seasons. PLoS One, 2017; 12: e0171734 
Eynon N, Hanson ED, Lucia A, Houweling PJ, Garton F, North KN, Bishop DJ. Genes for elite power and sprint performance: ACTN3 leads the way. Sport Med, 2013; 43: 803-817

Eynon N, Banting LK, Ruiz JR, Cieszczyk P, Dyatlov DA, Maciejewska-Karlowska A, Sawczuk M, Pushkarev VP, Kulikov LM, Pushkarev ED, Femia P, Stepto NK, Bishop DJ, Lucia A. ACTN3 R577X polymorphism and team-sport performance: A study involving three European cohorts. J Sci Med Sport, 2014; 17: 102-106

Fattahi Z, Najmabadi H. Prevalence of ACTN3 (the athlete gene) R577X polymorphism in Iranian population. Iran Red Crescent Med J, 2012; 14: 617-622

Holdys J, Krysciak J, Stanislawski D, Gronek P. Polymorphism of the alpha-ACTN3 gene in individuals practising different sports disciplines. Biol Sport, 2011; 28(2): 101-106

Houweling PJ, Berman YD, Turner N, Quinlan KGR, Seto JT, Yang N, Lek M, Macarthur DG, Cooney G, North $\mathrm{KN}$. Exploring the relationship between $\alpha$-actinin-3 deficiency and obesity in mice and humans. Int $J$ Obes (Lond), 2017; 41: 1154-1157

Ivarsson N, Westerblad H. Why Gene Loss Is an Evolutionary Gain. $\alpha$-Actinin-3: PLoS Genet, 2015; 11: e1004908

Joo $\mathrm{CH}$, Seo D. Analysis of physical fitness and technical skills of youth soccer players according to playing position. J Exerc Rehabil, 2016; 12: 548-552

Kalinowski P, Bojkowski Ł, Śliwowski R. Motor and psychological predispositions for playing football. TRENDS Sport Sci, 2019; 2(26): 51-54

Kikuchi N, Nakazato K. Effective utilization of genetic information for athletes and coaches: focus on ACTN3 R577X polymorphism. J Exerc Nutr Biochem, 2015; 19: 157-164

Kikuchi N, Miyamoto-Mikami E, Murakami H, Nakamura T, Min S-K, Mizuno M, Naito H, Miyachi M, Nakazato K, Fuku N. ACTN3 R577X genotype and athletic performance in a large cohort of Japanese athletes. Eur J Sport Sci, 2016; 16: 694-701

Kim H, Song K, Kim C. The ACTN3 R577X variant in sprint and strength performance. J Exerc Nutr Biochem, 2014; 18: 347-353

Loturco I, Pereira LA, Kobal R, Maldonado T, Piazzi AF, Bottino A, Kitamura K, Cal Abad CC, De Arruda M, Nakamura FY. Improving sprint performance in soccer: Effectiveness of jump squat and olympic push press exercises. PLoS One, 2016; 11: e0153958

Ma F, Yang Y, Li X, Zhou F, Gao C, Li M, Gao L. The Association of Sport Performance with ACE and ACTN3 Genetic Polymorphisms: A Systematic Review and Meta-Analysis. PLoS One, 2013; 8: e54685

MacArthur DG, North KN. A Genetic Influence on Muscle Function and Athletic Performance. Exerc Sport Sci Rev, 2007; 35: 30-34

MacArthur DG, Seto JT, Raftery JM, Quinlan KG, Huttley GA, Hook JW, Lemckert FA, Kee AJ, Edwards MR, Berman Y, Hardeman EC, Gunning PW, Easteal S, Yang N, North KN. Loss of ACTN3 gene function alters mouse muscle metabolism and shows evidence of positive selection in humans. Nat Genet, 2007; 39: 1261-1265

Mirkov DM, Kukolj M, Ugarkovic D, Koprivica VJ, Jaric S. Development of anthropometric and physical performance profiles of young elite male soccer players: a longitudinal study. J Strength Cond Res, 2010; 24: $2677-2682$

Newton RU, Kraemer WJ. Developing explosive muscle power: implications for a mixed methods training strategy. Strength Cond, 1994; 16: 20-31

Norman B, Esbjörnsson M, Rundqvist H, Osterlund T, von Walden F, Tesch PA. Strength, power, fiber types, and mRNA expression in trained men and women with different ACTN3 R577X genotypes. J Appl Physiol, 2009; 106: 959-965 
North K, Yang N, Wattanasirichaigoon D, Mills M, Easteal S, Beggs AH. A common nonsense mutation results in $\alpha$-actinin-3 deficiency in the general population. Nat Genet, 1999; 21: 353-354

Orysiak J, Busko K, Michalski R, Mazur-Różycka J, Gajewski J, Malczewska-Lenczowska J, Sitkowski D, Pokrywka A. Relationship between ACTN3 R577X polymorphism and maximal power output in elite Polish athletes. Medicina (Kaunas), 2014; 50: 303-308

Papadimitriou ID, Lucia A, Pitsiladis YP, Pushkarev VP, Dyatlov DA, Orekhov EF, Artioli GG, Guilherme JPLF, Lancha AH, Ginevičienė V, Cieszczyk P, Maciejewska-Karlowska A, Sawczuk M, Muniesa CA, Kouvatsi A, Massidda M, Calò CM, Garton F, Houweling PJ, Wang G, Austin K, Druzhevskaya AM, Astratenkova I V., Ahmetov II, Bishop DJ, North KN, Eynon N. ACTN3 R577X and ACE I/D gene variants influence performance in elite sprinters: a multi-cohort study. BMC Genomics, 2016; 17: 285

Pimenta EM, Coelho DB, Cruz IR, Morandi RF, Veneroso CE, De Azambuja Pussieldi G, Carvalho MRS, Silami-Garcia E, De Paz Fernández JA. The ACTN3 genotype in soccer players in response to acute eccentric training. Eur J Appl Physiol, 2012; 112: 1495-1503

Pimenta EM, Coelho DB, Veneroso CE, Barros Coelho EJ, Cruz IR, Morandi RF, De A Pussieldi G, Carvalho MRS, Garcia ES, De Paz Fernández JA. Effect of ACTN3 Gene on Strength and Endurance in Soccer Players. J Strength Cond Res, 2013; 27: 3286-3292

Radzimiński Ł, Rompa P, Dargiewicz R, Ignatiuk W, Jastrzębski Z. An Application of Incremental Running Test Results to Train Professional Soccer Players. Balt J Heal Phys Act, 2010; 2: 66-73

Santiago C, González-Freire M, Serratosa L, Morate FJ, Meyer T, Gómez-Gallego F, Lucia A. ACTN3 genotype in professional soccer players. Br J Sports Med, 2008; 42: 71-73

Silva JR, Nassis GP, Rebelo A. Strength training in soccer with a specific focus on highly trained players. Sport Med. Open, 2015; 1: 17

Szalata M, Słomski R, Balko S, Balko I. Advances in athlete genomics in 2019; short review. TRENDS Sport Sci, 2019; 2(26): 55-61

Vincent B, De Bock K, Ramaekers M, Van den Eede E, Van Leemputte M, Hespel P, Thomis MA. ACTN3 (R577X) genotype is associated with fiber type distribution. Physiol Genomics, 2007; 32: 58-63

Williams CA, Oliver JL, Faulkner J. Seasonal monitoring of sprint and jump performance in a soccer youth academy. Int J Sports Physiol Perform, 2011; 6: 264-275

Yang N, Macarthur DG, Gulbin JP, Hahn AG, Beggs AH, Easteal S, North K. ACTN3 Genotype Is Associated with Human Elite Athletic Performance. Am J Hum Genet, 2003; 73: 627-631

\section{Corresponding author:}

\section{Domańska-Senderowska}

Dept. of Molecular Bases of Medicine, Medical University of Lodz, Pomorska 251/C-5 Street, 92-213 Łódź, Poland,

E-mail: daria.domanska@umed.lodz.pl 